
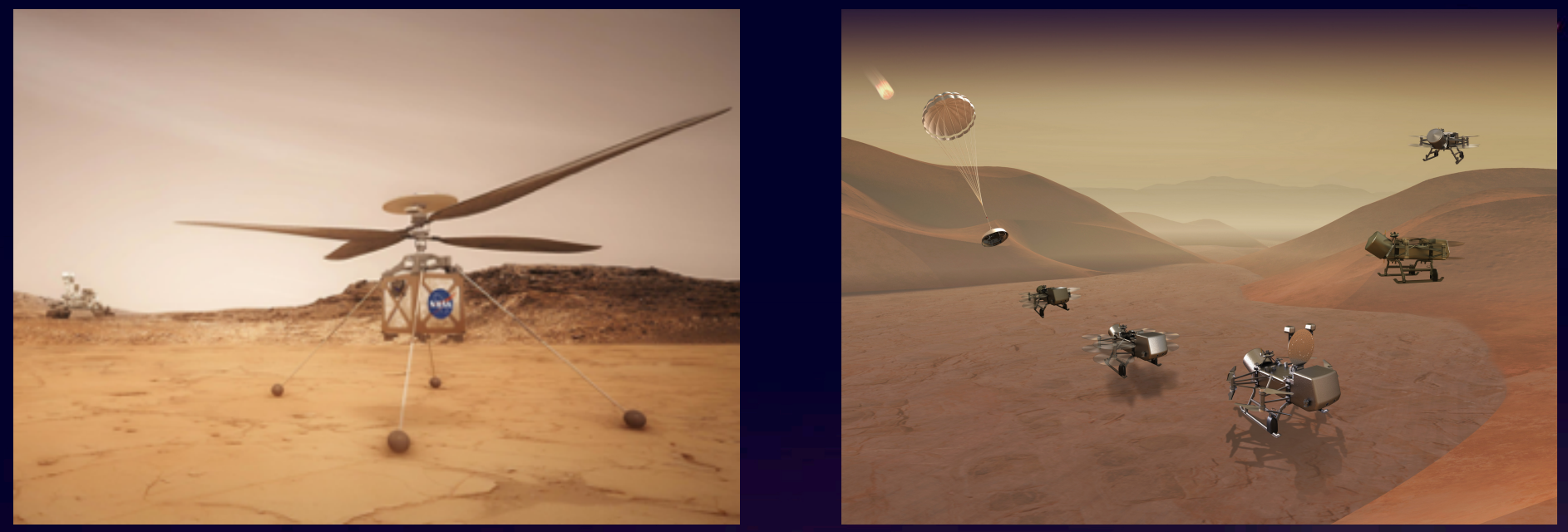

\title{
Space Drones: An Opportunity to Include, Engage, Accelerate, and Advance
}

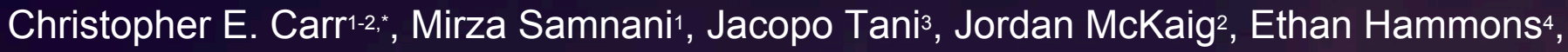
Dava J. Newman'4, Koki Ho1, Ariel Ekblaw5, Nathan Truelove 6

${ }^{1}$ Georgia Institute of Technology, Daniel Guggenheim School of Aerospace Engineering, Atlanta, GA, USA. ${ }^{2}$ Georgia Institute of Technology, School of Earth and Atmospheric Sciences, Atlanta, GA, USA. ${ }^{3}$ ETH Zurich, Institute for Dynamic Systems and Control, Zurich, Switzerland. ${ }^{4}$ MIT Department of Aeronautics and Astronautics, Cambridge, MA, USA. ${ }^{5 M I T}$ Media Lab Space Exploration Initiative, Cambridge, MA, USA. ${ }^{6}$ Monterey Bay Aquarium Research Institute, Moss Landing, California, USA.

*Primary author: cecarr@gatech.edu, +1-617-216-5012.

\section{Co-Signers}

Morgan L. Cable NASA Jet Propulsion Laboratory, California Institute of Technology Juergen Rauleder, Brian C. Gunter, E. Glenn Lightsey, Jonathan Rogers, and Mitchell Walker Georgia Institute of Technology, Daniel Guggenheim School of Aerospace Engineering, Atlanta, GA, USA

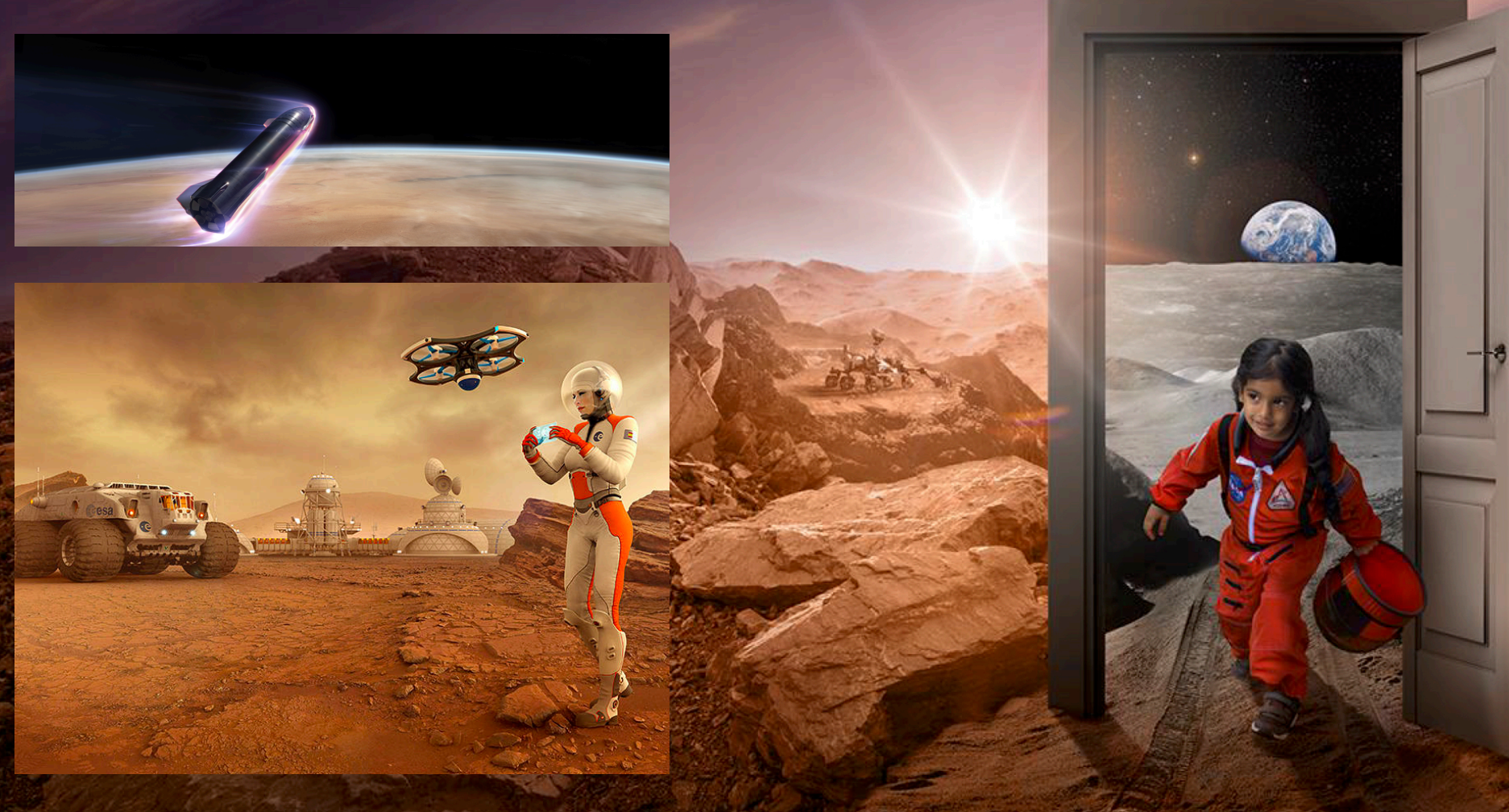




\section{MOTIVATION}

An autonomy revolution is underway on Earth, and autonomous vehicles are being employed in space to enable diverse missions including on Mars ${ }^{1}$, Titan ${ }^{2}$, and other Ocean Worlds.

Miniaturized sensors, hardware, novel materials and structures, additive manufacturing, machine learning, and other advancements are being integrated to enable capabilities once firmly in the realm of science fiction. Machine learning is being applied to predictive maintenance, trajectory optimization, generative designs, improved quality control, training, robot perception, autonomous decision making, and other areas.

Autonomous technology development has valuable applications for missions to a diverse array of planetary environments ${ }^{3}$, and has been identified as an area of key technology investment for the next generation of interplanetary missions ${ }^{4}$. Despite substantial progress, the challenges of planetary exploration, including seeking and supporting life beyond Earth, require new capabilities. These include, but are not limited to, accessing surface and subsurface regions unreachable by large rovers or humans, and operating with extreme efficiency and for sustained periods without communication. Addressing these complex challenges will require broad and persistent efforts, enabling opportunities to:

1. Accelerate the development and application of autonomy to space missions by leveraging a broad range of creativity, insight, expertise, and perseverance.

2. Meet the moral imperative of inclusivity, ensuring that NASA's mission", to "pioneer advances in aeronautics, space exploration, science, and technology to transform our understanding of the universe, unlock new opportunities, and inspire the world" is not only for the people but also by the people.

3. Engage individuals and give them agency to create an autonomous future on Earth and in space that represents the full breadth of our better selves.

4. Broaden participation in aerospace and space exploration through the integration of education and research, including enhanced participation in space missions.

Stakeholders in planetary science, and more broadly, robotic and human space exploration, should leverage and extend the autonomy revolution to enhance mission science return, our future in space, and human health and wellbeing. Addressing these challenges requires us to engage a global workforce, and create an environment where the seeds of ideas can become inventions that grow and propagate.

We believe that Space Drones, small uncrewed vehicles guided by remote or autonomous means, provide one compelling platform to achieve these ends. The feasibility of driving and flying on other worlds has now been demonstrated in the lab ${ }^{1}$, and will soon be demonstrated on the surface of another world ${ }^{1-2}$. With the helicopter Ingenuity now on its way to Mars, and the Dragonfly mission in development, the time is ripe to further develop a space autonomy ecosystem. This ecosystem is not new but can be strengthened by building upon existing programs and, where appropriate, creating new ones.

\section{CONTEXT}

The Road to Inclusivity 
Inclusion is a moral imperative and a well-documented path to performance ${ }^{6}$. This is especially true in the context of planetary science and astrobiology, where solutions require synthesis of multiple disciplines and perspectives. Whereas there have been field-specific improvements in diversity, in the geosciences, there has been little progress in ethnic and racial diversity in the last 40 years ${ }^{7}$. Furthermore, diversity by itself is inadequate and unsustainable; cultures must also change to engender inclusion and belonging ${ }^{8}$. One approach to educational inclusion is to change the problem framework whereas another is to change the norms: Duckietown, below, reframes autonomy in terms that conform less to traditional gender stereotypes; as an example, women doing robotics and leading space missions (GRAIL, Psyche, and Dragonfly, led by Maria T. Zuber, Lindy Elkins-Tanton, and Elizabeth Turtle, respectively) are changing the norms and serve as role models. These and other approaches can lead to cultural change. Recent efforts by NASA to add inclusion as a core value, support conference codes of conduct, and provide resources for grant writing, mission proposal development, career development, and new PIs are notable.

\section{Educational Paths to Enhancing the Space Autonomy Ecosystem}

NASA and other stakeholders operate many programs supporting advancement in science, technology, engineering, art, math, and design (STEAMD), in order to achieve goals including innovation in science and technology. These programs naturally overlap with the themes relevant to a space autonomy ecosystem. We describe a few examples.

Massively Open Online Courses (MOOCS) regularly exceed tens of thousands of enrollees for a single course. EdX has $14 \mathrm{M}$ learners and 50M registrations. Although completion rates are often low (5\%), shorter courses are completed at higher frequencies ${ }^{9}$. MOOCs enable inclusion by creating often-free educational experiences that can lead to certificates, but also exclude those without routine high-speed Internet access.

Aerial Robotics (https://www.coursera.org/learn/robotics-flight), a MOOC taught by Vijay Kumar (University of Pennsylvania) has 95k people enrolled as of the August 2020 start date. This and other courses use micro air vehicles to teach flight mechanics, control theory, trajectory planning, and integrate learning by doing.

Duckietown (https://www.duckietown.org/) is a state-of-the-art open-source ecosystem ${ }^{10}$ geared towards teaching autonomy using low-cost self-driving cars (duckiebots) and (duckie)drones, that function in a rubber-duckie populated smart urban environment, focusing on vision as means of perception. This playful approach to learning autonomy is geared towards multi-modal autonomous vehicle operations, and addresses challenges from the component to system levels, to open scientific questions in embodied $\mathrm{Al}^{11}$. These challenges can be varied to teach entry-level autonomy ${ }^{12}$ as well as pose research-grade problems ${ }^{13}$.

The NASA Community College Aerospace Scholars (NCAS) program, geared towards community college students, integrates a five-week online course with a four-day engineering workshop at a NASA center (https://nasaostem.okstate.edu/). It incorporates team building, networking, as well as an engineering design challenge (e.g., a rover competition), and has involved students from 141 Minority Serving Institutions (MSIs) to date.

\section{Competitions as a Driver of Innovation in the Space Autonomy Ecosystem}

Recent efforts to drive innovation through competition include the XPrize and MIT Solve. NASA and other organizations also conduct a diverse set of competitions oriented around different 
aerospace or exploration themes including disciplines, themed or multi-theme hackathons, and integrative efforts such as vehicle challenges (underwater, aerial, surface, human rover).

These competitions (Figure 1) foster innovation directly and indirectly, by allowing individuals to get excited, build their personal and professional network, develop skills, envision career paths, meet mentors, experience the thrill of accomplishment and, critically, fail quickly, and often with low stakes. These are examples and the list of competitions above is not inclusive. Many competitions are built around Earth-based themes and could be adapted to incorporate "Space Drone" themes either as an option (e.g., Space Apps) or as an annual topic (e.g., Vertical Flight Society). Many other relevant activities exist including analog missions ${ }^{14}$.

\begin{tabular}{|c|c|}
\hline Domain & Competition \\
\hline All & $\begin{array}{l}\text { The NASA Space Apps hackathon (https://www.spaceappschallenge.org/), a global weekend event now in its } 9 \text { th year, } \\
\text { attracts tens of thousands of participants annually ( } 29,000 \text { participants, } 225 \text { events, } 71 \text { countries in } 2019 \text { ). This event is great for } \\
\text { team building, ideation, and pilot projects/prototyping, it does not allow enough time to develop deep expertise or robust } \\
\text { solutions. In principle, anyone can join, without any requirement of being part of an academic program or part of an engineering } \\
\text { society, although there are other barriers to entry including the location of events, time commitment required, and other factors. }\end{array}$ \\
\hline Land & $\begin{array}{l}\text { FIRST Robotics Competition (https://www.firstinspires.org/) allows for high school students to get engaging STEM and } \\
\text { teamwork experience through an annual themed robotics competition. In } 2019 \text {, the theme was "Destination: Deep Space" where } \\
\text { the challenge was to build a robot that could load as much cargo as possible before a rocket takes off. }\end{array}$ \\
\hline & $\begin{array}{l}\text { The MATE International ROV Competition (https://materovcompetition.org/) challenges students from across the U.S. and } \\
\text { around the world to engineer ROVs to complete a set of mission tasks based on real-world, workplace scenarios. }\end{array}$ \\
\hline Sea & $\begin{array}{l}\text { RoboSub (https://robosub.org/) is an international student competition where student teams from around the world design and } \\
\text { build robotic submarines(AUV) which mimic operations of real-world systems, currently deployed around the world for } \\
\text { underwater exploration, seafloor mapping, sonar localization, and other missions. }\end{array}$ \\
\hline \multirow{3}{*}{ Air } & $\begin{array}{l}\text { The Association for Unmanned Aerial Vehicle Systems International (https://www.auvsi-suas.org/) competition requires } \\
\text { students to design, integrate, report on, and demonstrate a uncrewed aerial system capable of autonomous flight and navigation, } \\
\text { remote sensing, and execution of a specific set of tasks. }\end{array}$ \\
\hline & $\begin{array}{l}\text { The International Aerial Robotics Competition challenges students with a high level of difficulty } \\
\text { (http://www.aerialroboticscompetition.org/). At each event, participants move aerial robotics forward by attempting to solve } \\
\text { significant and useful mission challenges in earth's atmosphere. }\end{array}$ \\
\hline & $\begin{array}{l}\text { The Vertical Flight Society competition, now in its } 38 \text { th year, has a different theme each year (https://vtol.org/awards-and- } \\
\text { contests/student-design-competition), with rotating sponsors including Bell Helicopter, Sikorsky Aircraft, the U.S. Army } \\
\text { Research Lab, Airbus, Leonardo Helicopters, and The Boeing Company. }\end{array}$ \\
\hline \multirow{2}{*}{ Space } & $\begin{array}{l}\text { The NASA Human Exploration Rover Challenge (https:/www.nasa.gov/stem/roverchallenge/competition/index.html), } \\
\text { prior to } 2014 \text { referred to as the Great Moonbuggy Race, is an annual competition for high school and college students to design, } \\
\text { build, and race human-powered, collapsible vehicles over simulated lunar/Martian terrain. }\end{array}$ \\
\hline & $\begin{array}{l}\text { The Mars Society's University Rover Challenge (http://urc.marssociety.org/) encourages students to build remotely } \\
\text { operated rovers that can accomplish a variety of tasks that might one day assist astronauts working on the surface of Mars at the } \\
\text { Mars Society's Mars Desert Research Station (MDRS) near Hanksville, Utah, USA. }\end{array}$ \\
\hline
\end{tabular}

Figure 1. Competitions used to drive innovation in different vehicle domains.

\section{Student and Public Participation in Space Missions}

Aerospace and Planetary Science degree programs often incorporate space mission design in senior undergraduate or graduate courses. The Planetary Science Summer School (PSSS) at Caltech/JPL (https://pscischool.jpl.nasa.gov/), geared towards doctoral students through junior faculty, provides a hypothesis-driven mission design experience intended to develop future space mission leaders ${ }^{15}$. Students and the public have participated in space missions directly by requesting imaging of Mars via the HiWish program ${ }^{16}$ as well as controlling a camera onboard the GRAIL spacecraft orbiting the moon via the MoonKam program ${ }^{17}$.

NASA engages students in space missions through full life cycle (conceive, design, implement, operate) opportunities including stratospheric balloon flights (e.g., LSU's High Altitude Student Payload program), sounding rockets, and CubeSats. Student-developed payloads are represented in multiple space missions. For example, the Regolith X-ray Imaging Spectrometer (REXIS) ${ }^{18}$ on the OSIRIS-REx mission is student-run, with the goal to help teach the next generation how to design and create spaceflight hardware. 


\section{RECOMMENDATIONS}

Accelerate the development and application of autonomy to space missions

- Support both mission-capability driven development and blue-sky open-ended work.

- Evaluate augmentation of existing NASA R\&D programs to facilitate additional development of small vehicle autonomy including drones.

- Leverage contributions from all stakeholders, including industry.

- Provide explicit opportunities linked to the integration of robotic space exploration and human exploration and habitation on the moon and Mars.

- Provide reference designs to frame research in specific areas.

- Build Space Drone themes into existing competitions where appropriate.

- Consider the development of new programs including a Space Drones pipeline that could involve short-term MOOCs and a new focused competition.

- Provided platforms for competitions and research on Space Drones, including thermal vacuum chamber(s), Mars analog testbeds, and high altitude balloons.

- Ensure that all non-ITAR data related to flight operations beyond Earth (e.g., Ingenuity and Dragonfly) are available through the NASA Planetary Data System and/or via alternatives (e.g., GitHub, Thingiverse) to facilitate use by any stakeholders.

Meet the moral imperative of inclusivity

- Cast a wide net; engage a global community in Space Drone activities and include such activities as part of existing (outreach, STEM, career development) programs.

- Provide subsidized access to reduce socioeconomic barriers; avoid imposing unnecessary (e.g., degree, society) barriers.

- Engage with minority serving institutions (MSIs), Historically Black Colleges and Universities (HBCUs), and technical colleges, including with financial support.

- See the position paper Diversity in action: Solutions for a more diverse and inclusive decade of planetary science and astrobiology for additional recommendations.

Engage individuals and give them agency

- Support many entry points into the space autonomy ecosystem, including across the entire educational spectrum.

- Build an array of opportunities, across different time scales, so that individuals can benefit from, and contribute to, the space autonomy ecosystem over a sustained period. Examples include a MOOC, hackathons, student project/research support via SpaceGrant or other mechanisms, graduate funding via FINESSE, and other mechanisms.

- Provide a web of continuity between individuals, teams, and opportunities, so that people can navigate from one experience to another; common transition pathways should be evaluated and supported through existing or new mechanisms.

\section{Broaden participation in space exploration}

- Provide payload opportunities as part of NASA and industry-related space activities.

- Include "Space Drone" opportunities as an explicit part of ongoing planning for combined human and robotic exploration on the Moon.

- Include "Space Drone" opportunities as part of human Mars exploration robotic precursor missions extending through human exploration and habitation on Mars. 


\section{Pipeline Enhancement}

Massively Open Online Course. We envision a Space Drone themed MOOC that could help participants learn relevant skills, and carry out projects related to future capability needs assessment, technology evaluation, skill development, and teambuilding. A short course ( 5 weeks) may help support higher completion rates than a long course. Strategic skill enhancement and team building are likely more critical than comprehensive coverage, and the MOOC could help develop domain-specific skills as a feeder to existing or new competition(s).

Extending Existing Competitions: Many options exist; here we mention two possibilities. NASA selects many topics for its annual Space Apps hackathon and could further incorporate space autonomy to facilitate ideation and team building. A Mars or Titan drone theme could be selected for a future Vertical Flight Society (VFS) competition. Competitions could be aligned with Mars 2020 activities as Perseverance is slated to land on Mars in February 2021.

Space Drones Competition: We envision a new competition that would include both domain-specific and integrated interdisciplinary challenges. Teams could be constructed on an open basis or have institutional, international or national team sub-competitions (think Robot Olympics: the 2021 Olympics will have a strong robotics component). Sub-competitions could also require specific types of cross-group collaboration, such as a system built by multiple teams across geographic or international boundaries, as is common in space instruments and missions.

Taking the example of a Mars micro-rover or helicopter, a competition could include separate sub-competitions focused on structural optimization, obstacle avoidance, path optimization, energy storage or transfer, rotor design, packability and deployment, sampling (accessing special regions), planetary protection (sterilization, sample handoff), autonomous mission planning, sensor technologies (imaging, ice prospecting, weather), mapping, facility monitoring, steep slope access, and other application-specific goals that would support robotic and human exploration on the surface of Mars. Metrics used to evaluate each sub-competition could include mass, energy, power, volume, time, accuracy, and other relevant measures.

Aside from these domain-specific activities, integrative challenges could be utilized in a similar way as by the DARPA robotics challenge (2012-2015). NASA operates simulated planetary landscapes (e.g., JPL Mars Yard I \& II, JSC Rock Yard), to facilitate research and testing of payloads. Related facilities are used in NASA competitions such as the NASA Human Exploration Rover Challenge. JPL and other organizations also have thermal vacuum chambers that can be used to simulate Mars surface conditions, where flying is extremely challenging due to the low atmospheric pressure ( $7 \mathrm{mbar}$ ), similar to stratospheric conditions on Earth.

Competitions may utilize physical or virtual environments and modes of participation. The ongoing pandemic has highlighted both limitations and benefits of virtual events. One approach to integrating physical hardware and virtual events is to send kits to participants or have competition entries sent to a central testing location; code/simulations can be run remotely. Simulations utilizing a probability distribution (e.g., Monte Carlo) to represent uncertain parameters (e.g., landscape, atmosphere), would help to provide more accurate measures of system performance.

Stratospheric Balloon Model for Mars Rotorcraft. Stratospheric balloons are routinely used to deploy science payloads and elevate the technology readiness level (TRL) in preparation for space missions. Stratospheric testing of Mars rotorcraft technologies is a logical next step after ground-based thermal vacuum chamber testing due to Mars-like pressures, and in some cases, temperatures. Deployment and recovery strategies would need to be refined. 


\section{Mission Participation}

Drones on the Moon. At the MIT150 Symposium on Earth, Air, Ocean and Space: The Future of Exploration symposium, Jennifer Buz presented SatellitePlay, a concept for gamified robotic lunar exploration that envisioned anyone on Earth with a video game controller and internet access being able to drive micro-vehicles on the lunar surface. The video game market now exceeds \$150B/year (2019). We envision a "sandbox" where micro-rovers can be utilized for educational and research purposes. The benefits of such a program should be evaluated for inclusion in Project Artemis as one approach to integrating robotic and human exploration.

Human Mars Exploration. SpaceX is currently developing Starship, with a projected Mars landing capability of 100 tons (91,000 kg or 100 Perseverance rovers). There are many steps to successfully landing such a vehicle on Mars, yet SpaceX, BlueOrigin, and others are making significant progress towards delivering significant mass to the surface of the Moon, Mars, or other worlds. We envision robotic or human missions bringing micro-drones or improved versions of Ingenuity (see Mars Science Helicopter position paper).

A fleet of small vehicles could integrate STEAMD activities across academia, industry, students, and the public, creating a Mars "sandbox" for autonomy. The fleet could map the nearby rock distribution to centimeter-scale, ground-truth ice and other potential resources, serve as a network of weather sensors, and validate technologies such as autonomous operations, 5G/high-speed mesh network communication, wireless power delivery, and develop new capabilities. Access to (candidate) special regions (regions where life as we know it might be capable of reproducing) could leverage highly sterile micro vehicles for sampling and transport of samples to a clean yet accessible location, facilitating acquisition by a larger rover or human, "breaking the chain" and reducing the risk of forward contamination. Such a platform would help advance space autonomy at the agent and fleet level.

\section{IMPACTS}

We seek to democratize access to space, and through this process, unleash new space mission capabilities. Developing a strong pipeline, beginning as early as elementary school, is key to the health of the fields of autonomy and machine learning ${ }^{19}$. We recommend longitudinal tracking of impact on participants including in comparison to a control group, such as done by the FIRST Robotics Competition. This approach supplements enrollment and completion data and has provided strong evidence of impact (FIRST female alumni are 5X "more likely to declare majors in engineering and computer science than their peers"). Other measures include research experience, doctoral program enrollment/completion, fellowships, and mission participation. Self-assessment of skills, confidence, professional network, and measures of belonging can be used in combination to assess the impact of inclusion efforts ${ }^{20}$. Assessment should also include technology-centered metrics such as publications, grants, patents, licensing, citations, downloads of research products, and (code) forks.

A robust space autonomy ecosystem will also facilitate pathways between space and non-space applications, and benefit stakeholders including participating individuals, academia, government, and industry. Expanding opportunities within this ecosystem, including across international borders, can help to rebuild a pro-science reputation of the U.S. and improve collaboration with our international partners. 
Figure 2. Selected space mission capabilities enabled by a robust space autonomy ecosystem.

By developing a robust space autonomy ecosystem now, we are investing in a future full of new space mission capabilities (Figure 2).

Advances may arise

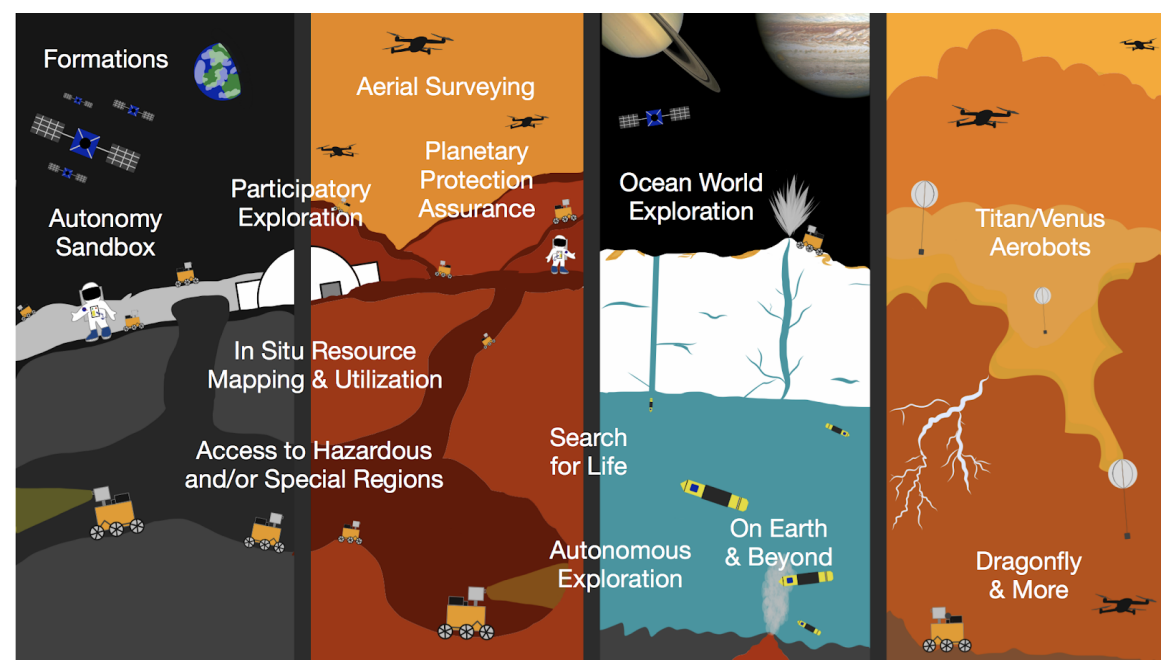
directly from early-stage programs and emerge from later activities of participants. Through incremental and transformational change, we can achieve a future where autonomous vehicles drive, swim, or fly longer, further, more autonomously, and cooperatively. We will expand access to steep slopes, fresh impact craters, caves, and other challenging terrains of geological and astrobiological interest, as well as providing valuable mapping, monitoring, and surveying capabilities to support crewed activities on celestial bodies such as the Moon and Mars. Potential impacts described by other position papers include Mars surface relay networks, a Mars Science Helicopter, aerial surveying of Mars remnant magnetism, volcanic cave exploration as a priority for astrobiology, planetary protection for human Mars missions, aerial platforms for Venus, autonomy for Ocean World exploration, and operations concepts for asteroids and comets. Building an inclusive space autonomy ecosystem is a critical enabler of exploring the cosmos as well as seeking and supporting life beyond Earth.

\section{REFERENCES}

[1] Balaram et al. (2018) AIAA Atmospheric Flight Mechanics Conference. [2] Lorenz et al. (2018) Johns Hopkins APL Technical Digest 34(3), 374-87. [3] [NAS 25168] NAS (2018) "Autonomy on Land and Sea and in the Air and Space" [4] [NAS 25186] NAS (2018) "Visions into Voyages for Planetary Science in the Decade 2013-2022: A Midterm Review" [5] NASA Science Strategy (2020) "SCIENCE 2020-2024: A Vision for Scientific Excellence" [6] Bourke and Dillon (2018) "The diversity and inclusion revolution: Eight powerful truths" Deloitte Review, issue 22. [7] Bernard and Cooperdock (2018) Nature Geosci, 11(5):292-5. [8] AGU Diversity and Inclusion Strategic Plan (2018). [9] K. Jordan (2014) IRRODL, 15(1):133-60. [10] Paull et al. (2017) IEEE ICRA doi: 10.1109/ICRA.2017.7989179. [11] Zilly et al. (2020) The NeurIPS'18 Competition, Springer, Cham. doi: 10.1007/978-3-030-29135-8_3. [12] Brand et al. (2018) IEEE/RSJ IROS doi: 10.1109/IROS.2018.8593943. [13] Tani et al. (2020) IEEE/RSJ IROS (accepted), https://ttic.edu/ripl/publications/tani20.pdf. [14] NAS (2020) "Advancing Aerial Mobility: A National Blueprint" [15] Budney et al. (2017) LPSC XLVIII 2828. [16] Chojnacki et al. (2020) LPSC LI 2095. [17] Zuber et al. (2012) Space Sci Rev, 82(2):3-24 doi:10.1007/s11214-012-9952-7. [18] Masterson et al. (2018) Space Sci Rev, 214(1)1-26. [19] NAS (2018) "The Frontiers of Machine Learning" [20] NAS (2019) "The Science of Effective Mentorship in STEMM". NAS: National Academy of Sciences. 